Meta

Journal des traducteurs

Translators' Journal

\title{
L'étude des langues des spécialités médicales : un scialytique sur un champ opératoire
}

\section{Amal Jammal}

Volume 35, numéro 1, mars 1990

Actes du colloque international « La traduction proligère "

URI : https://id.erudit.org/iderudit/003243ar

DOI : https://doi.org/10.7202/003243ar

Aller au sommaire du numéro

Éditeur(s)

Les Presses de l'Université de Montréal

ISSN

0026-0452 (imprimé)

1492-1421 (numérique)

Découvrir la revue

Citer cet article

Jammal, A. (1990). L'étude des langues des spécialités médicales : un scialytique sur un champ opératoire. Meta, 35(1), 50-54. https://doi.org/10.7202/003243ar 


\section{L'ÉTUDE DES LANGUES DES SPÉCIALITÉS MÉDICALES: UN SCIALYTIQUE SUR UN CHAMP OPÉRATOIRE}

AMAL JAMMAL

Université de Montréal, Montréal, Canada

Je ne parlerai ici que des langues des spécialités médicales parce qu'elles me sont plus familières que d'autres. Je suis néanmoins convaincue que ce que j'en dirai pourrait s'appliquer à toute langue de spécialité.

Comme on le sait, le scialytique (du grec «skia = ombre» et «luein = dissoudre») est l'appareil d'éclairage qui surplombe le champ opératoire pour en supprimer les ombres portées. Or, dans toute langue de spécialité - et la langue médicale n'échappe pas à la règle - il existe des zones grises, des ombres portées, qui brouillent non seulement les dénominations de certaines notions, mais parfois aussi les descripteurs mêmes de ces notions.

L'étude des langues de spécialité oblige le terminologue à disséquer tout terme qui lui paraît suspect ou toute définition qui lui semble nébuleuse. Elle lui permet ainsi de mettre au jour les anomalies qui nuisent à la clarté de l'énoncé ou qui peuvent donner naissance, par une analogie structurelle abusive, à certaines interprétations erronées. De plus, il peut lui arriver de constater qu'un même terme a, d'un dictionnaire à l'autre ou d'un manuel à l'autre, une définition différente et, plus grave encore comme nous le verrons, une expression mathématique différente. Et cela sans que la divergence ne soit explicitée ni même mentionnée dans ces ouvrages. Ou encore, il peut constater que l'ombre est parfois importée (comme on dit de l'inflation qu'elle est importée), nous venant directement de l'anglais.

Ces zones d'ombre peuvent donc avoir plusieurs origines. L'une d'elles peut être une confusion dans les descripteurs de la notion que l'on veut désigner. Je donnerai comme exemple un terme appartenant à la langue de l'allergie. Il s'agit d'une expression que les auteurs francophones empruntent telle quelle à l'anglais en la mettant entre guillemets, pour bien indiquer qu'il s'agit d'un emprunt et cela, faute de pouvoir lui trouver un équivalent français honorable. L'expression en question est «prick test». Permettez-moi d'expliquer brièvement ce qu'est le «prick test». Il s'agit d'un test permettant de diagnostiquer l'hypersensibilité immédiate à un allergène donné. La méthode consiste à placer, sur la peau, une goutte d'une solution contenant l'allergène et de piquer la peau, à travers cette goutte, pour y faire pénétrer l'allergène. On attend ensuite une dizaine de minutes et l'on observe ce qui se passe. De prime abord, on peut se demander où est le problème et pourquoi l'emprunt? Il doit sûrement exister un dictionnaire crédible qui donnerait l'équivalent français de ce terme. Et de fait, dans le Gladstone, un bon dictionnaire médical d'équivalences, nous trouvons «prick test $=$ test intradermique ${ }^{1} \gg$. Néanmoins, une simple vérification, comme est tenu d'en faire tout traducteur ou tout terminologue qui se respecte, révèle de fortes divergences avec d'autres dictionnaires tout aussi crédibles. En réalité, à en croire le Manuila ${ }^{2}$ et le Flammarion ${ }^{3}$, le terme français test intradermique, proposé par Gladstone, recouvrirait plutôt la notion d'intradermoréaction et serait donc 
l'équivalent d'intradermal test et non de prick test, ce qui semble d'ailleurs plus logique. Devant ces contradictions, notre terminologue poursuit ses recherches, rassemble un nombre important de contextes relevés dans des manuels d'allergie fiables, recherche les définitions proposées par les autres dictionnaires médicaux reconnus, pour procéder enfin à des recoupements et à des comparaisons entre les divers descripteurs et ainsi tirer la chose au clair. Là, il s'aperçoit que le problème est beaucoup plus compliqué qu'il n'y paraissait et que les anomalies ne sont pas seulement terminologiques mais qu'elles sont aussi notionnelles.

Notionnelles, car d'un manuel à l'autre, la description que l'on fait du «prick test» n'est pas la même. Pour certains, en effet, il s'agit d'une piqûre qui doit être effectuée dans l'épiderme. Et ce détail, très important, est bien spécifié, soit explicitement ${ }^{4}$, soit implicitement lorsque l'auteur précise qu'il faut piquer la peau sans la faire saigner. (L'on sait en effet que seul l'épiderme n'est pas vascularisé; le derme, qui l'est abondamment, saigne infailliblement dès qu'on le pique.) Donc, notre terminologue a la confirmation que, dans le «prick test», il faut piquer l'épiderme. Néanmoins, il se trouve d'autres auteurs pour préciser qu'il faut piquer... le derme ${ }^{6}$. Donc, première ombre au tableau.

Mais il y a mieux encore: pour certains, il faut piquer la peau à l'aide d'une microlance $^{7}$; pour d'autres... il faut la scarifier ${ }^{8}$, c'est-à-dire l'inciser superficiellement! Comme on le voit, la notion elle-même n'est pas claire. Et l'ombre est aussi dense du côté français que du côté anglais. Mais là où les francophones ont la palme, c'est, comme on le verra, dans les désignations mêmes des tests de l'allergie.

En effet, pour démêler l'écheveau terminologique, il a fallu placer le «prick test» dans la série des autres tests de l'allergie, afin d'arriver, en procédant par comparaison ou par analogie, à le nommer en français. Il s'est alors avéré que nornbre d'auteurs francophones confondent, terminologiquement, les tests de l'allergie... et leurs résultats ! C'est ainsi que l'on peut lire dans le Dictionnaire français de médecine et de biologie, de Manuila et coll. ${ }^{9}$, que test épicutané a comme synonyme épidermoréaction. On peut également constater que cuti-réaction est utilisé dans des manuels et des dictionnaires français pour désigner ce que les anglophones appellent scratch test ${ }^{10}$. En somme, si un test est négatif, on pourrait avoir une cuti-réaction sans réaction! Par ailleurs, le terme cuti-réaction, tel qu'il est utilisé, est de plus une confusion entre le générique et le spécifique. En effet, cuti signifiant peau, toute réaction de la peau, à quelque niveau qu'elle ait lieu - épiderme, derme ou hypoderme - est une cuti-réaction. Or, ce terme est réservé, sans justification aucune, à la seule scarification de l'épiderme.

Comme on le voit, le bobo ici est multifactoriel. Quelle serait la solution dans pareil cas? On pourrait proposer d'abord de définir sans équivoque la notion elle-même, en l'occurrence le "prick test», en faisant la lumière, par des recherches et des vérifications, sur la méthode qu'il implique*. Dans le cas qui nous occupe, on précisera donc qu'il s'agit d'une piqûre - et non d'une scarification - faite dans l'épiderme - et non dans le derme. Ensuite, on peut décider, en suivant le modèle terminologique anglais, de nommer tous les tests français en fonction de leur méthode respective ou de la couche de la peau qu'ils intéressent. C'est alors que le «prick test» prendra tout naturellement sa place dans la série des autres tests et pourra se débarrasser de son allure étrangère et de ses guillemets. On aurait en effet, en allant de l'extérieur vers l'intérieur:

- le test épicutané, ainsi nommé parce qu'on applique sur la surface de la peau un carré de toile portant la substance allergène. Il aurait comme équivalent anglais patch test;

- le test de la scarification épidermique, qui implique une scarification de l'épiderme. Là, s'ajoute le descripteur «méthode» pour le différencier du prick test qui, lui aussi, a lieu dans l'épiderme (équivalent anglais : scratch test); 
- le test de la piqûre épidermique pour désigner le prick test, et enfin

- le test intradermique, pour désigner l'intradermal test.

Voilà pour la confusion attribuable à des brouillages d'origine à la fois notionnelle et terminologique.

Mais l'ombre peut aussi être due à une fausse monosémie. Et là, je proposerai l'exemple de l'indice thérapeutique. Lorsqu'un traducteur doublé d'un biochimiste m'a téléphoné pour me demander ce qu'était, à mon avis, l'indice thérapeutique d'un médicament, je suis tombée à pieds joints dans le piège et lui ai sorti ma définition, que je croyais imparable. L'indice thérapeutique? Mais voyons ! C'est la dose efficace moyenne (que certains appellent la dose curative moyenne) rapportée à la dose toxique moyenne. En fait, c'est la mesure de l'innocuité d'un médicament. Autrement dit, si je veux mesurer le facteur d'innocuité de l'aspirine utilisée comme analgésique, je calculerai sa dose efficace moyenne (celle qui soulage la douleur) rapportée à sa dose toxique moyenne (celle qui provoque l'apparition des signes de toxicité). Je pourrais avoir par exemple :

$650 \mathrm{mg}$ (dose efficace moyenne)

$2000 \mathrm{mg}$ (dose toxique moyenne).

Il va sans dire que plus l'écart entre le numérateur et le dénominateur est grand, moins le médicament est jugé nocif. Or, mon interlocuteur m'affirme, me donnant la référence idoine, en l'occurrence le Goodman et Gilman ${ }^{11}$ qui est la bible en pharmacologie, que l'indice thérapeutique y est défini comme la dose efficace moyenne rapportée à la dose létale moyenne. On conviendra que la différence est de taille. À titre indicatif : nous avions tout à l'heure, pour l'aspirine,

$$
\frac{650}{2000}
$$

là nous aurions

$$
\frac{650}{8000 ? 10000 ?}
$$

Je ne sais exactement au bout de combien de comprimés on passe l'arme à gauche, mais je sais que le dénominateur n'est plus du tout le même. Et ensuite, comment peut-on déterminer la dose létale? Par autopsie? Très douteux, puisque pour incriminer l'aspirine avec certitude, il faut administrer le médicament, jusqu'à ce que mort s'ensuive, à un individu par ailleurs sain. Et encore! Pour déterminer la dose létale moyenne, il faudrait avoir la possibilité de la mesurer chez une importante série de cadavres! Ce sont ces deux aspects qui nous ont mis la puce à l'oreille et orienté nos recherches dans un autre sens. Nous avons pu ainsi découvrir que l'indice thérapeutique se mesure différemment selon le contexte dans lequel il est utilisé. Dans un contexte de médecine clinique, on pourra mesurer la dose efficace sur la dose toxique, que l'on déterminera par l'apparition des effets toxiques. Dans un contexte d'études de laboratoire, par contre, là où l'on peut se permettre de provoquer la mort de l'animal, on a la possibilité d'aller jusqu'à la dose létale. Autrement dit, l'indice thérapeutique devrait avoir non pas une mais bien deux définitions, exprimées par deux rapports différents, selon le contexte d'utilisation; une polysémie qui n'est spécifiée dans aucun dictionnaire, d'où la confusion.

Une troisième source d'ombre pourrait être de nature sémasiologique, due à une similitude sémantique trompeuse entre deux expressions dont les définitions sont pourtant bien différentes. L'exemple cette fois est pris dans la langue de l'épidémiologie et les 
deux expressions incriminées sont risque attribuable à un facteur donné et fraction étiologique du risque. À cause de la parenté de sens entre le mot «attribuable» et le mot «étiologique», ces deux expressions peuvent paraître identiques. Certains épidémiologistes, et des plus chevronnés, les ont prises l'une pour l'autre, attribuant à la deuxième le sens de la première ${ }^{12}$. Et pourtant, encore une fois, cette similitude ne résiste pas à l'analyse et surtout pas à la mise en forme mathématique des deux expressions. En effet, le risque attribuable à un facteur donné est la différence entre le taux d'incidence d'une maladie au sein d'une population exposée à un facteur donné et le taux d'incidence de cette maladie au sein de la population non exposée. Autrement dit, si je veux calculer le risque du cancer du poumon attribuable à la cigarette, je calcule le taux d'incidence du cancer dans une population de fumeurs, moins le taux d'incidence dans une population de non-fumeurs. Le risque attribuable à la cigarette pourrait être par exemple :

$$
\frac{30}{1000} \text { (fumeurs) }-\frac{5}{1000} \text { (non fumeurs) }=\frac{25}{1000} \text {, soit } \mathbf{0 , 0 2 5}
$$

La fraction étiologique du risque par contre est la différence entre le taux d'incidence dans une population de fumeurs et le taux d'incidence dans une population de nonfumeurs, mais cette fois rapportée au taux d'incidence dans la population de fumeurs. Ce serait donc:

$$
\frac{\frac{30}{1000} \text { (fumeurs) }-\frac{5}{1000} \text { (non fumeurs) }}{\frac{30}{1000}}=\mathbf{0 , 8 3}
$$

Les chiffres sont éloquents, les deux expressions sont loin d'être interchangeables.

Et je pourrais citer d'autres cas de turbulence terminologique: confusion entre dépistage multiple et dépistage multiphasique, confusion probablement imputable à la monosémie anglaise ${ }^{13}$, tout à fait incompréhensible d'ailleurs: le dépistage multiple désigne un dépistage dans lequel, par mesure d'économie sans doute, on procède au dépistage de plus d'une maladie ou de plus d'un facteur de risque (l'hypertension, l'hyperglycémie, etc.), alors que le dépistage multiphasique peut ne s'intéresser qu'à une seule maladie, mais s'effectuer en plusieurs phases. Ou encore confusion entre le taux de mortalité et le taux de létalité. Mortalité et létalité étant, dans la langue courante, deux termes voisins, on est tenté de confondre les deux taux. En réalité, il s'agit de deux paramètres tout à fait différents, puisque le taux de mortalité par le sida, par exemple, est le nombre de décès dus au Sida, rapporté à la population générale, - disons 25/100 000 - alors que le taux de létalité du sida est le nombre de décès dus au sida rapporté aux personnes atteintes du sida, soit par exemple 25/50. Encore une fois, le dénominateur et par conséquent le taux ne sont plus du tout les mêmes.

Ainsi donc, celui qui étudie une langue de spécialité, surtout d'une spécialité jeune, risque de rencontrer des zones grises auxquelles il ne peut se permettre de passer outre et qui ne sont pas seulement de nature terminologique, peu s'en faut, mais aussi de nature notionnelle, ce qui rend l'ombre autrement plus dense et plus difficile à dissiper. Aussi devra-t-il, afin de cerner ces zones grises et d'en trouver l'origine, disséquer, fouiller, comparer, consulter. Mais s'il aime son métier, il n'aura de cesse que lorsqu'il aura réussi à rendre clairs et les champs sémantiques des notions et les termes par lesquels on les désigne, en les débarrassant, comme le ferait un scialytique, de toute ombre portée qui pourrait en fausser la signification. 


\section{Références}

1. GLADSTONE, W. J. (1984): Dictionnaire anglais-français des sciences médicales et paramédicales, $2^{\mathrm{e}}$ éd., Saint-Hyacinthe, Québec, Edisem, et Paris, Maloine, p. 743.

2. MANUILA, A., L. MANUILA, M. Nicole et H. LAMBERT (1971): Dictionnaire français de médecine et de biologie, Paris, Masson, tome II, p. 534.

3. Dictionnaire de médecine Flammarion (1989), $3^{\mathrm{e}}$ éd., Paris, Flammarion Médecine-Sciences, p. 448.

4. LESSOR, M. H., T. H., LEE and D. M. KEMENY (1987) : Allergy: An International Textbook, Baltimore, Williams F. Wilkins, p. 281.

5. JACKSON, W. F. et R. CERIO (1987) : Color Atlas of Allergy, Schering Corporation, p. 17.

6. BLACQUE-BÉLAIR, B. Mathieu de FOSSEY et M. FOURESTIER, Dictionnaire des constantes biologiques et physiques, $5^{\mathrm{e}}$ éd., Paris, Maloine, p. 826.

7. JACKSON, W. F. et R. Cerio, op. cit., p. 17.

8. MICHEL, François-Bernard et Jean BOUSQUET (1986): Les Allergies, la fin d'une énigme, Paris, Les Guides Hachette.

9. MANUILA et coll. (1972), op. cit., tome 3, p. 930.

10. Grand Dictionnaire encyclopédique Larousse (1982), Paris, p. 2851.

11. Goodman \& Gilman's Pharmacological Basis of Therapeutics (1985), A. G. Gilamn et al., New York, MacMillan, 7 th ed., p. 37

12. RUMEAU-ROUQUETTE, C., G. BRÉART et R. PADIEU (1981): Méthodes en épidémiologie, $2^{\mathrm{e}}$ éd., Paris, Flammarion Médecine-Sciences, p. 274.

13. Dorland's Illustrated Medical Dictionary (1981) : W.B. Saunders, Philadelphia, 26th ed., p. 1183

* Pour plus de détails concernant la démarche terminologique, voir META, 34-4, décembre 1989. 